Journal of Antimicrobial Chemotherapy (1989) 23, 179-188

\title{
Natural methicillin resistance in comparison with that selected by in-vitro drug exposure in Staphylococcus aureus
}

\author{
B. Berger-Bächl", A. Strässle and F. H. Kayser \\ Institute of Medical Microbiology, University of Zürich, Postfach, CH 8028 Zürich, \\ Switzerland
}

\begin{abstract}
Methicillin resistant staphylococci selected during serial passage in increasing concentrations of methicillin were compared with an isogenic methicillin resistant strain carrying the methicillin resistance determinant (mec) found in clinical isolates of Staphylococcus aureus. Selection for methicillin resistance was followed by changes in the binding characteristic of penicillin binding proteins (PBP3) 2 and 4 . There was no immunological nor genetic relationship between the mec-determined low affinity PBP2' and the PBPs of the in-vitro selected mutants.

The Tn551 insertion $\Omega 2003$, which inactivates mec determined methicillin resistance, but which is not linked to mec, also partially reduced in-vitro selected methicillin resistance. This suggests that this methicillin resistance controlling factor contributes at least partially to methicillin resistance selected in vitro.
\end{abstract}

\section{Introduction}

Methicillin resistance in clinical isolates of Staphylococcus aureus is associated with the production of a supplementary penicillin-binding protein, designated PBP2' or PBP2a (Hartman \& Tomasz, 1984; Reynolds \& Fuller, 1986; for a review see Lyon \& Skurray, $1987)$ that has low affinity for $\beta$-lactam antibiotics. The structural gene for PBP2' has been cloned (Matsuhashi et al., 1987) and sequenced (Song et al., 1987). It is located on the methicillin resistance determinant mec (unpublished results). Mec maps at a specific chromosomal site (Kuhl, Pattee \& Baldwin, 1978) in methicillin resistant staphylococei (MRSA). PBP2' is thought to be the only functional PBP at concentrations of $\beta$-lactam antibiotics sufficient to kill sensitive cells (Reynolds \& Brown, 1985). The amount of PBP2' in cells is, however, not related to the level of resistance (Berger-Bāchi, Strässle \& Kayser, 1986; Chambers \& Hackbarth, 1987; Madiraju, Brunner \& Wilkinson, 1987). At least one further factor, which is not linked to mec, controls the expression of methicillin resistance (Berger-Bächi, 1983; Berger-Bächi \& Kohler, 1983; Hartman \& Tomasz, 1986; Kornblum et al., 1986). One such factor maps on the chromosome near thrA (Berger-Bächi \& Kohler, 1983). The nature and mechanism of action of this second factor, is not yet known. The Tn551 insertion $\Omega 2003$ there however abolishes mec-determined methicillin resistance but nevertheless allows production of PBP2' in normal amounts (Berger-Bächi et al., 1986).

Methicillin resistant strains can also be selected in vitro by serial passage of sensitive strains on increasing concentrations of methicillin. Such methicillin resistant strains show homogeneous resistance, in contrast to the heterogeneous resistance displayed by 
most clinical isolates (Kayser et al., 1971). Changes in the binding capacity of their PBPs have been observed (Tonin \& Tomasz, 1986). Although not clinically relevant, such in-vitro selected strains might help to understand the additional factor(s) that control mec.

An intriguing question is whether clinically $\mathrm{Mc}^{r}$ strains showing heterogeneous resistance use an additional resistance mechanism related to the one used by the in-vitro selected strains.

\section{Materials and methods}

\section{Strains and culture conditions}

The strains of $S$. aureus used in this study are listed in Table I. They were grown at $37^{\circ} \mathrm{C}$ in L-broth $(10 \mathrm{~g} / \mathrm{l}$ tryptone (Difco, USA), $5 \mathrm{~g} / 1$ yeast extract (Difco, USA), $5 \mathrm{~g} / 1$ $\mathrm{NaCl}$ ) where not otherwise mentioned. Population analysis was made by plating aliquots of an overnight culture on L-agar plates containing increasing amounts of methicillin (Beecham Pharmaceuticals, USA). Minimal inhibitory concentrations (MICs) of antibiotics were determined by microbroth dilution according to the recommendations of the National Committee for Clinical Laboratory Standards (National Committee for Clinical Laboratory Standards, 1985). Transductions of the chromosomal insertion $\Omega 2003$ (chr::Tn551) were made with the generalized transducing phage $80 \alpha$, then selecting for transductants on agar containing $20 \mathrm{mg} / 1$ of erythromycin.

Table I. Bacterial strains used in this study

\begin{tabular}{|c|c|c|c|}
\hline Strain & Relevant genotype & $\begin{array}{l}\text { Relevant } \\
\text { phenotype }\end{array}$ & Origin/reference \\
\hline $\begin{array}{l}\text { BB255 } \\
\text { BB255-P1-M1 } \\
\text { BB255-P2-M3.5 } \\
\text { BB255-P3-M11 } \\
\text { BB255-P4-M26 } \\
\text { BB255-P5-M54 } \\
\text { BB255-P6-M90 } \\
\text { FK394 } \\
\text { FK394-P6-M84 } \\
\text { BB270 } \\
\text { BB308 } \\
\text { BB577 } \\
\text { BB578 }\end{array}$ & $\begin{array}{l}\text { NCTC8325 } \\
\text { NCTC8325 } \\
\text { NCTC8325 } \\
\text { NCTC8325 } \\
\text { NCTC8325 } \\
\text { NCTC8325 } \\
\text { NCTC8325 } \\
\text { NCTC8325-4 } \\
\text { NCTC8325-4 } \\
\text { NCTC8325 mec } \\
\text { NCTC8325 mec } \\
\Omega 2003 \text { (chr: : Tn55l) } \\
\text { NCTC8325 } \\
\Omega 2003 \text { (chr: : Tn55l) } \\
\text { NCTC8325 } \\
\Omega 2003 \text { chr: : Tm55l) }\end{array}$ & $\begin{array}{l}\mathrm{Mc}^{\mathrm{s}} \\
\mathrm{Mc}^{\mathrm{r}} \\
\mathrm{Mc}^{\mathrm{r}} \\
\mathrm{Mc}^{\mathrm{r}} \\
\mathrm{Mc}^{\mathrm{r}} \\
\mathrm{Mc}^{\mathrm{r}} \\
\mathrm{Mc}^{\mathrm{r}} \\
\mathrm{Mc}^{\mathrm{s}} \\
\mathrm{Mc^{3 }} \\
\mathrm{Mc}^{\mathrm{r}} \\
\mathrm{Mc}^{\mathrm{E}} \mathrm{Er} \\
\mathrm{Mc}^{\mathrm{r}} \mathrm{Er} \\
\mathrm{Mc}^{\mathrm{s}} \mathrm{Er}\end{array}$ & $\begin{array}{l}\text { Berger-Bächi (1983) } \\
\text { this study } \\
\text { this study" } \\
\text { this study" } \\
\text { this study" } \\
\text { this study } \\
\text { this study } \\
\text { this study } \\
\text { Berger-Bächi (1983) } \\
\text { Berger-Bächi (1983) } \\
\text { this studye } \\
\text { this study }\end{array}$ \\
\hline
\end{tabular}

Mutant derived from strain BB25S during selection for growth on increasing concentrations of methicillin as described in Materials and methods.

Mutant derived from strain FK 394 as in ".

Err transductant of strain BB255-P6-M90.

'Er' traneductant of strain BB255-P5-M54.

Mc', Methicillin sensitive; Mc' methicillin resistant; Er'; erythromycin resistant. 


\section{In-vitro selection of methicillin resistant mutants}

Overnight cultures $10^{10} \mathrm{cfu}$ of either strain BB255 or strain FK394 were spread on BHI agar (Difco, USA) containing increasing amounts of methicillin and were incubated at $35^{\circ} \mathrm{C}$. A single colony growing at the highest concentration was isolated and kept for further analysis. The same procedure was repeated with this mutant using higher methicillin concentrations. Each mutant thus obtained was named by the number of the step when it was isolated (P1-P6) and by the concentration of methicillin (mg/l) M1-M90 in the plate from which it was isolated.

\section{Penicillin-binding proteins}

Isolation of cell membranes, labelling with $\left({ }^{3} \mathrm{H}\right)$-benzylpenicillin-ethylpiperidinium salt $25 \mathrm{Ci} / \mathrm{mmol}$ (Merck, USA), competition assays with nafcillin (Bristol Laboratories, USA), and separation of the penicillin binding proteins (PBPs) on SDS polyacrylamide gels, as well as staining by Coomassie brilliant blue and detection of the PBPs by fluorography was as described earlier (Berger-Bāchi et al., 1986). Partial proteolytic digests of labelled PBPs with SV8 protease and separation of the labelled polypeptides was by the method described by Reynolds \& Fuller (1986).

\section{DNA techniques}

Isolation of chromosomal DNA, digestion of the DNA by restriction endonucleases, separation of the DNA on agarose gels, transfer to nitrocellulose membranes, hybridization with $\left({ }^{32} \mathrm{P}\right)$-labelled DNA probes and detection on $\mathrm{X}$-ray films was as described earlier (Berger-Bächi et al., 1986).

\section{Results}

\section{Physiological characterization of the methicillin-resistant mutant strains}

Methicillin-resistant mutants could be isolated from sensitive strains of $S$. aureus and were able to grow on plates containing up to $90 \mathrm{mg} / \mathrm{l}$ methicillin. Attempts to isolate mutants beyond this concentration failed owing to spontaneous lysis of the colonies on plates with higher methicillin concentrations.

The mutants were stable upon storage or growth on non-selective media. The generation time of the highest resistant mutant strain BB255-P6-M90 was significantly longer $(67 \mathrm{~min})$ than that of the parent strain BB255 (45 min) when growing on non-selective medium at $30^{\circ} \mathrm{C}$.

The changes in the minimum inhibitory concentrations (MICs) of some representative $\beta$-lactams for the resistant mutants are shown in Figure 1. The first step in methicillin resistance brought the largest increase in MIC values. Ampicillin, amoxycillin, piperacillin as well as nafcillin and oxacillin behaved in a similar manner to penicillin and methicillin, data for which are shown in Figure 1(a). Acquisition of resistance to the first and second generation cephalosporins is illustrated in Figure 1(b) for cephalothin and cefamandole. Similar behaviour was noted for cefazolin and cefuroxime (not shown). With the third generation cephalosporins there was a smaller increase in resistance. They are represented in Figure $1(b)$ by cefotaxime and similar 


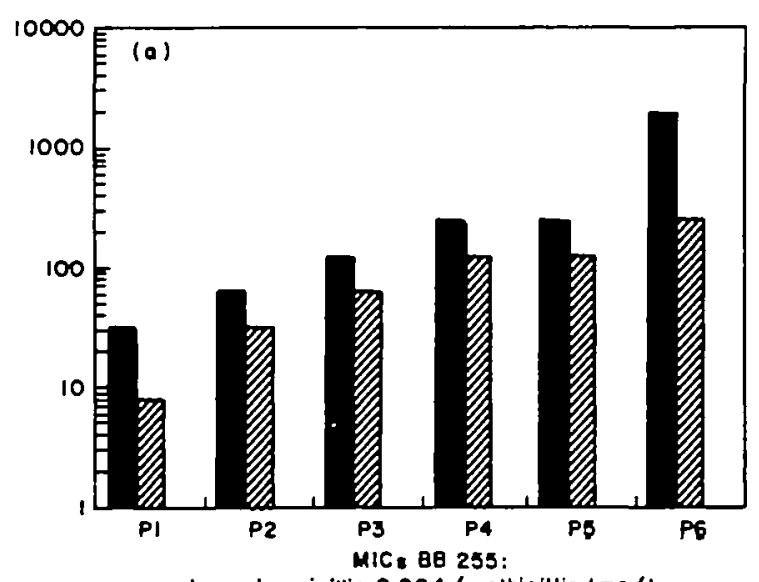

benzyl ponicillin 0-004/methlcillin $1 \mathrm{mg} / \mathrm{l}$

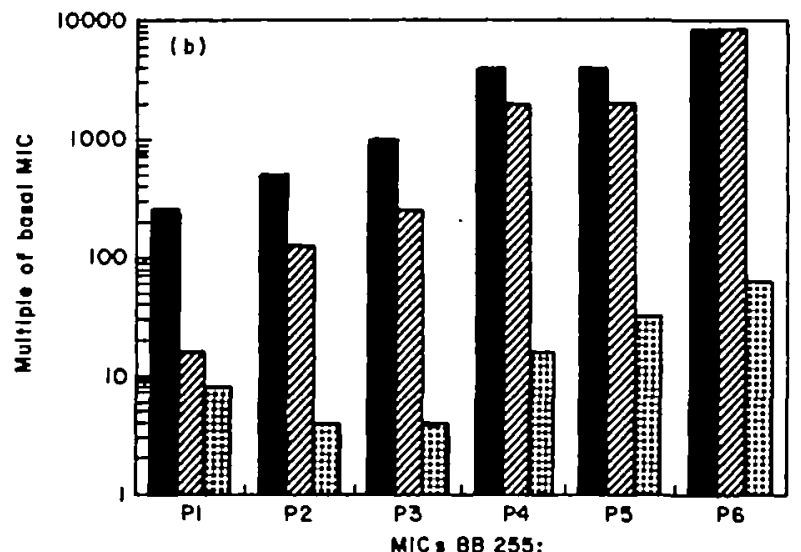

cephalothin $0-008 /$ cofamondole $0-03 /$ cofotox/me $0.5 \mathrm{mo} / \mathrm{l}$



Figure 1. Increase of the MIC of different $\beta$-lactam antibiotic for the methicillin resistant mutants selected from strain BB255. The MIC of each antibiotic against the sensitive strain BB255 is given at the bottom of the appropriate diagram. The value of one is arbitrarily assigned to this basal MIC. In the diagram the multiples of the basal MIC attained by the mutants is plotted for each step. P1, Strain BB255-P1-M1; P2, train BB255-P2-M3.5; P3, strain BB255-P3-M11; P4, strain BB255-P4-M26; P5, strain BB255-P5-M54; P6, strain BB255-P6-M90. (a) $\square$, Penicillin G; $\square$, methicillin; (b) $\square$, cephalotin; $\square$, cefamandole; $\mathbb{B}$, cefotaxime; (c) D, imipenem; M, cefaclor, $\mathbf{E}$, latamoxef. 

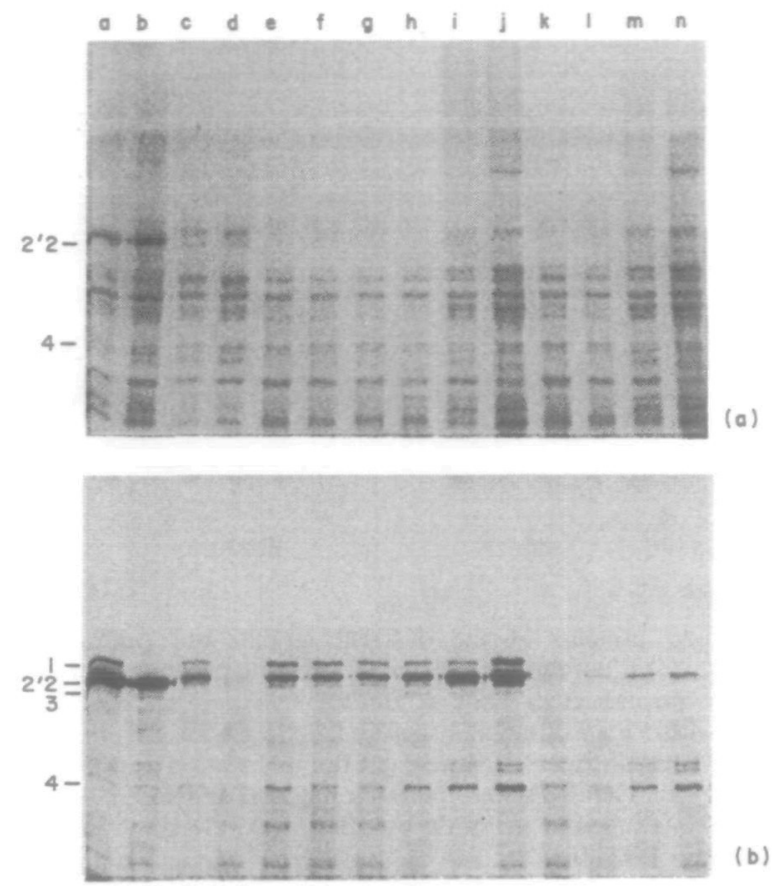

Flgure 2 Membrane proteins and PBP's of the different methicillin-resistant mutants. (a) Coomassie blue stained gel of membrane proteins of the various strains, prepared as described in Materiais and methods. The positions of PBP2, PBP2' and PBP4 ape indicated. (b) Fluorograph of ('H)-penicillin labelled penicillin binding proteins of the gel shown above. PBP's were labelled with $\left({ }^{3} \mathrm{H}\right)$-penicillin as described in Materials and methods. In competition experfments membranes were preineubated with $5 \mathrm{mg} / \mathrm{l}$ nafcillin before labelling. Lanes a, b, Strain BB270 (mec); c, d, strain BB255 (methicillin sensitive): a, c penicillin binding proteins without competition with nafacillin; b, d, after competition with $5 \mathrm{mg} / \mathrm{nafcillin}$. e, $k$, Strain BB255-P1-M1; f, strain BB255-P2-M3.5; 1 , strain BB255-P3-M11; h, strain BB255-P4-M26; $i$, m, strain BB255-P5-M54; j, n, strain BB255-P6-M90. e, f, g, h, i, j, Penicillin-binding proteins without competition with nafcillin; $\mathrm{k}, \mathrm{l}, \mathrm{m}, \mathrm{n}$, after competition with $5 \mathrm{mg} / \mathrm{nafcillin}$. The positions of the five PBPt are indicated.

behaviour was observed to ceftriaxone and ceftazidime. There was no increase in resistance to latamoxef (Figure 1(c)). For some antibiotics (e.g. cefaclor and imipenem Figure 1(c)) no further increase in resistance was seen after the first step. Mutants selected from strain FK394 gave comparable susceptibility patterns.

\section{Penicillin-binding proteins}

The gain in resistance to methicillin in the mutants was paralleled by an increase in the amount of PBP2 and PBP4 that was labelled with $\left({ }^{3} \mathrm{H}\right)$-penicillin (Figure 2(b)); however no increase in intensity of the Coomassie blue stained bands was seen at this position in the gel (Figure 2(a)). Lesser amounts stained proteins in the size range of PBP1 to PBP3 (80-70 kdal) were found in membrane preparations from the mutants. Other stepwise changes in the protein pattern of the mutants can be seen in Figure 2(a) but these did not involve the penicillin-binding proteins (Figure 2(b)). In competition experiments with nafcillin, concentrations that saturated all PBPs of sensitive strain BB255 (Figure 2(b) lane d), were not sufficient to saturate PBP2 and PBP4 of the mutants (Figure 2(b), lanes $k, 1, m$ and $n$ ). Thus, these PBPs had a lower 


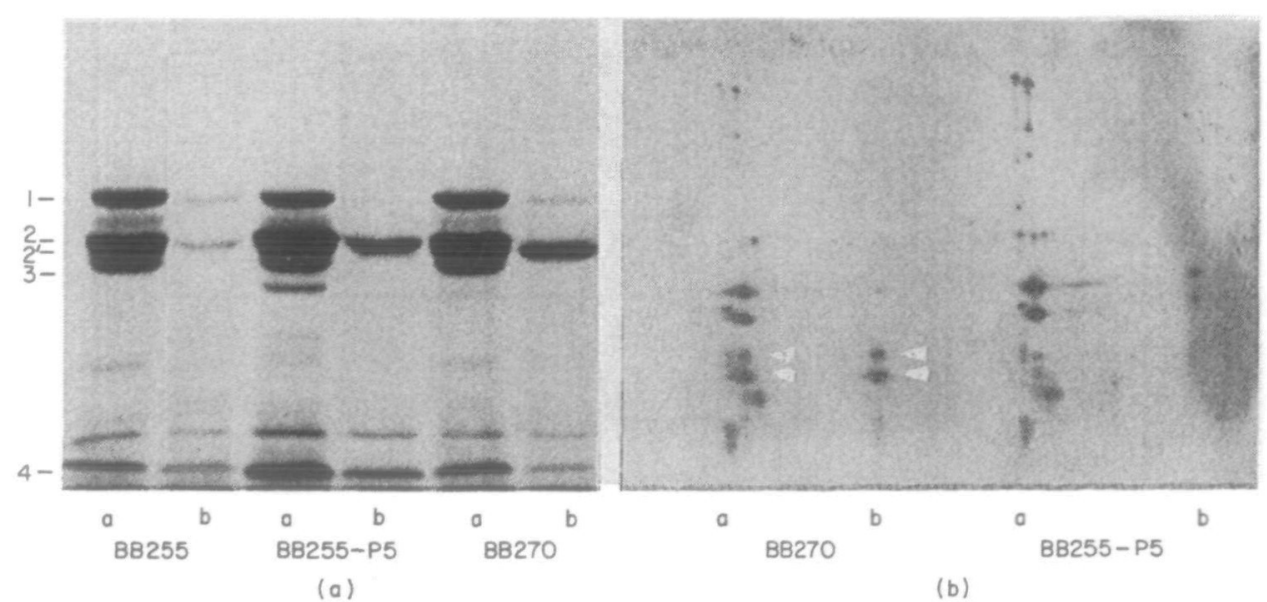

Fyore 3. Penicillin binding proteins of the different strains and penicilloyl-peptides after partial proteolysis with SV8 protease. (a) Fluorograph of penicillin-binding proteins separated on a SDS-PAGE gel. The positions of the PBP's are indicated. Lane a, PBPs labelled with $\left({ }^{3} \mathrm{H}\right)$-penicillin; b; the membrane extracts were preincubated with $5 \mathrm{mg} / \mathrm{l}$ of nafcillin before labelling with $\left({ }^{3} \mathrm{H}\right.$ )-penicillin. (b) Fluorograph of the penicilloyl-peptides. The lanes of Figure 3(a) were cut out and run on a second dimension. SV8 protease was included in the stacking gel. The PBPs were partially digested and the resulting labelled peptides were separated, as described in Materials and methods. Arrotw mark the positions of characteristic peptides produced by PBP2' from strain BB270.

affinity for nafcillin. They could still be labelled by $\left({ }^{3} \mathrm{H}\right)$-penicillin (Figure $2(\mathrm{~b})$ and 3(a). Similar behaviour was shown also by the low affinity, mec-specific PBP2' from the methicillin-resistant wild type strain BB270 (Figure 2(b), lane b). Nevertheless the reisistant PBP2 from strain BB255-P5-M54 migrated at the same position as the original PBP2 from sensitive strain BB255, which was distinct from the position of PBP2' (Figure 3(a)).

Partial proteolytic digests with SV8 protease of the labelled PBP's of the laboratory selected strain BB255-P5-M54 and of natural methicillin resistant strain BB270 carrying the mec determinant were compared (Figure 3(b)). The labelled peptides stemming from PBP2' are indicated by arrows. They were identified in competition experiments using nafcillin at concentrations sufficient to saturate all PBP's except PBP2'. They were only present in strain BB270 (Figure 3(b), lanes a and b). Peptides of different mobilities which were not saturated by the same nafcillin concentrations appeared in the in-vitro $\mathrm{Mc}^{\mathrm{r}}$ strain BB255-P5-M54 (Figure 3, lane b). These peptides migrated identically to those produced from PBP2 of the sensitive strain BB255.

There were no significant changes in the affinity of the PBPs of the mutant strains towards $\left({ }^{3} \mathrm{H}\right.$ )-penicillin compared with those of the sensitive parental strain (not shown).

\section{Genetic analysis of the mutants}

Attempts to transfer the methicillin resistance of strain BB255-P6-M90 to the sensitive strain BB255 by phage $80 \alpha$ transduction failed. Transductants grew only at the lowest level for selection of methicillin resistant mutants, where they could not be distinguished from spontaneous mutants. 


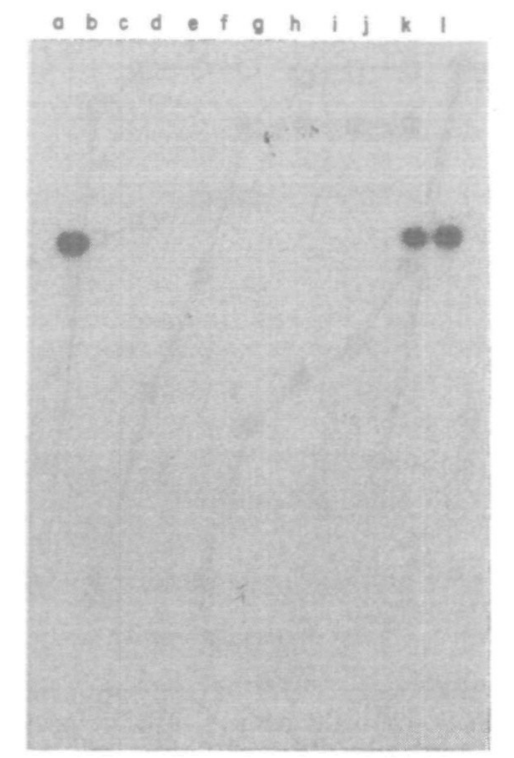

Fipure 4. Hybridization of a PBP2' DNA probe to EcoRI restricted chromosomal DNA of various strains. EcoRI digests of the chromosomal DNA of the different strains were separated on a gel and hybridized to a $2.2 \mathrm{~kb} X$ Xbal-HindllI probe containing the structural gene for PBP2' of strain BB270. Lanes a, $k$, strain BB270; b, strain BB255; c, strain BB255-P1-M1; d, strain BB255-P2-M3.5; e, strain BB255-P3-M11; f, strain BB255-P4-M26; g, strain BB255-PS-M54; h, strain BB255-P6-M90; i, strain BB578; $j$, strain BB577, and l, strain BB308.

There was no DNA homology between the structural gene for the mec specific, low affinity PBP2' and the chromosomal DNA of the laboratory selected mutants, as shown in Figure 4. Only the methicillin resistant strain BB270 and mutant strain BB308, which both contain the mec-resistance determinant, showed hybridization.

\section{Influence of $\Omega 2003$ (chr: : Tn551) on the mutants}

Insertion of $\Omega 2003$ is known to inactivate the mec-determined methicillin resistance, without affecting the PBP2' content of the cells (Berger-Bāchi et al., 1986). To test whether this regulating factor also affected in-vitro selected resistance, the chromosomal Tn551 insertion $\Omega 2003$ was transduced by phage $80 \alpha$ into strains BB255-P5-M54 and BB255-P6-M90, with selection for erythromycin resistance. All erythromycin-resistant transductants were found still to be methicillin resistant. They grew on plates containing $10 \mathrm{mg} / 1$ of methicillin, whereas strain BB308, an isogenic derivative of BB255, carrying mec as well as $\Omega 2003$ (chr.: Tn55l) was unable to grow at this methicillin concentrations. Closer analysis however showed that the MIC's of methicillin for transductants of BB255-P5-M54 and BB255-P6-M90 were reduced from 32 to $2 \mathrm{mg} /$, and from 128 to $8 \mathrm{mg} / 1$, respectively. The population analysis (Figure 5) showed that insertion $\Omega 2003$ (chr: : Tn55I) reduced the resistance level of the in-vitro selected strain BB255-P6-M90, but not as dramatically as in strain BB308 carrying the mec resistance determinant. In BB255, an example of an antibiotic sensitive $S$. aureus strain with a wild type background, $\Omega 2003$ (chr: : Tn55l) had no influence on antibiotic susceptibility. 


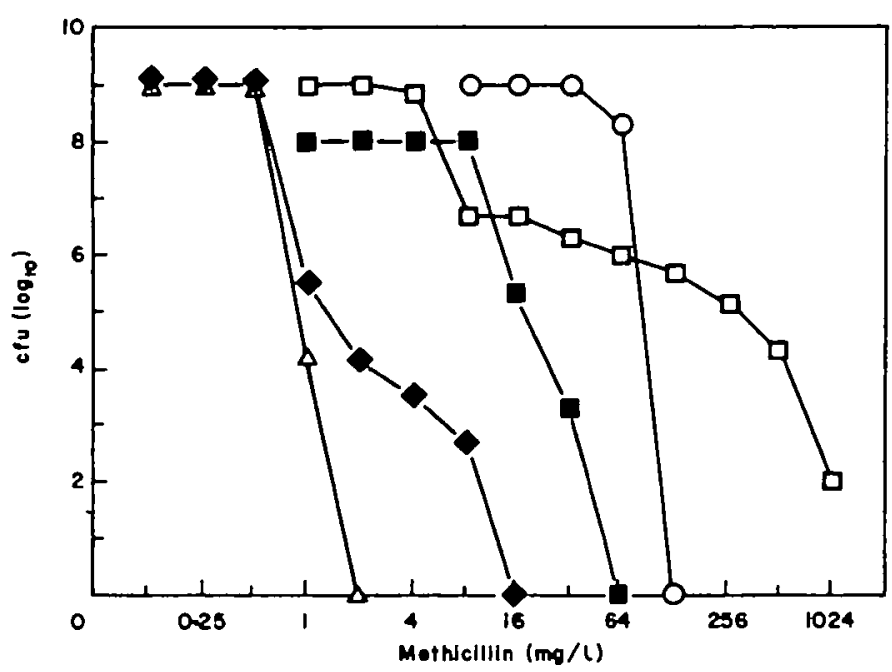

Flgure 5. Influence of insertion $\$ 2003$ (chr: : TnSSI) on the resistance levels for populations of different methicillin-resistant strains. Aliquots of overnight cultures of the different strains were plated on increasing concentrations of methicillin and the colony forming units were determined after $48 \mathrm{~h}$ incubation at $35^{\circ} \mathrm{C}$. $\triangle$, Methicillin sensitive strain BB255; $\square$, methicillin resistant strain BB270 (mec); $\bullet$, methicillin sensitive strain BB308, the control strain carrying mec and insertion $\mathbf{2 0 0 3}$ (chr.: Tn55I); O, methicillin resistant mutant BB255-P6-M90; $\square$, transductant strain BB577 derived from BB255-P6-M90 carrying insertion $\Omega 2003$ (chr. : TnSSI).

\section{Discussion}

Methicillin resistance acquired during selection is probably caused by multiple mutations at different loci on the chromosome. These change amongst other, unidentified factors, the affinity for $\beta$-lactam-antibiotics of PBPs 2 and 4 , and reduced the growth rate. The order and nature of the single step mutations leading to higher methicillin resistance depends probably on the strain chosen. Tonin \& Tomasz (1986) observed only a four-fold increase in the MIC of cefotaxime for their methicillin-resistant mutant M100 selected by methicillin, whereas mutant strains BB255-P6-M90 and FK394-P6-M84 both showed a 64-fold increase in the MIC of cefotaxime. Both sensitive strains used in this study were from the same parent, differing only in their prophage content. They responded in a similar way to selection pressure with increased methicillin concentrations.

There was no genetic relationship at the DNA level between the structural gene for PBP2' and the PBPs from the mutant strains. Nor did monoclonal antibodies directed towards PBP2' from $S$. aureus bind to membrane preparations of the mutant strains (P. Reynolds, personal communication). It is therefore unlikely that PBP2' from methicillin resistant staphylococci carrying mec has evolved by a series of point mutations within PBP2, as suggested by Tonin \& Tomasz (1986). The gene for mec-specific PBP2' might rather be of foreign origin (Reynolds, 1985).

No significant changes in the PBP affinity for $\left({ }^{3} \mathrm{H}\right)$-penicillin were observed in the mutant strains. Experimental conditions, however, could not rule out the presence of a mutated, lower affinity, PBP masked by the wild type form of PBP migrating at the same position in the gel. It appeared that the sensitive strain BB255 produced two forms of PBP2 of slightly different mobilities, distinguishable only by loading small 
amounts of membrane preparations on the gel (Figure 2, lane c). The mutant PBP2 might stem from one of the forms, that changed its affinity by point mutations, or was produced now in much larger amounts.

The Tn551 insertion $\mathbf{\Omega 2 0 0 3}$ that inactivates mec determined methicillin resistance, had a small effect also on laboratory-selected methicillin-resistant strains. This suggests that the mec controlling factor has also a more general function in resistance to $\beta$-lactams and is at least partially involved in the resistance mechanism of the laboratory mutants.

\section{Acknowledgements}

This work was supported by the Swiss National Science Foundation, grant 3.326-0.86. A. Strässle was supported by a grant from Hoffman-La Roche, and also in part by the Bonizzi Theler Stiftung. We thank Merck, Sharp \& Dohme for generous gifts of $\left({ }^{3} \mathrm{H}\right)$-benzylpenicillin. We are grateful for the collaboration of $P$. Reynolds (Cambridge) who tested our strains with monoclonal antibodies, and to C. Ryffel for the DNA probe for PBP2'.

\section{References}

Berger-Băchi, B. (1983). Insertional inactivation of staphylococcal methicillin resistance by Tn551. Journal of Bacteriology 154, 479-87.

Berger-Bächi, B. \& Kohler, M. L. (1983). A novel site on the chromosome of Staphylococcus aureus influencing the level of methicillin resistance: genetic mapping. FEMS Microbiology Letters 20, 305-9.

Berger-Bächi, B., Strässle, A. \& Kayser, F. H. (1986). Characterization of an isogenic set of methicillin-resistant and susceptible mutants of Staphylococcus aureus. European Journal of Clinical Micrabiology 5, 697-701.

Chambers, H. F. \& Hackbarth, C. J. (1987). Effect of $\mathrm{NaCl}$ and nafcillin on penicillin-binding protein 2 a and heterogeneous expression of methicillin resistance in Staphylococcus aureus. Antimicrobial Agents and Chemotherapy 31, 1982-8.

Hartman, B. J. \& Tomasz, A. (1984). Low-affinity penicillin-binding protein associated with $\beta$-lactam resistance in Staphylococcus aureus. Journal of Bacteriology 158, 513-6.

Hartman, B. J. \& Tomasz, A. (1986). Expression of methicillin resistance in heterogeneous strains of Staphylococcus aureus. Antimicrobial Agents and Chemotherapy 29, 85-92.

Kayser, F. H., Benner, E. J., Troy, R. \& Hoeprich, P. D. (1971). Experimental and clinical aspects of resistance determinants. Mode of resistance against beta-lactam antibiotics in staphylococci. Annals of the New York Academy of Sciences 182, 106-17.

Kornblum, J., Hartman, B. J., Novick, R. P. \& Tomasz, A. (1986). Conversion of a homogeneously methicillin-resistant strain of Staphylococcus aureus to heterogeneous resistance by Tn551-mediated insertional inactivation. European Journal of Clinical Microbiology 5, 714-8.

Kuhl, S. A., Pattee, P. A. \& Baldwin, J. N. (1978). Chromosomal map location of the methicillin resistance determinant in Staphylococcus aureus. Journal of Bacteriology 135, 460-5.

Lyon, B. R. \& Skurray, R. (1987). Antimicrobial resistance of Staphylococcus aureus: genetic basis. Microbiological Reviews 51, 88-134.

Madiraju, M. V. V. S., Brunner, D. P. \& Wilkinson, B. J. (1987). Effects of temperature, $\mathrm{NaCl}$, and methicillin on penicillin-binding proteins, growth, peptidoglycan synthesis, and autolysis in methicillin-resistant Staphylococcus aureus. Antimicrabial Agents and Chemotherapy 31, 1727-33.

Matsuhashi, M., Song, M. D., Ishino, F., Wachi, M., Doi, M., Inoue, M. et al. (1986). Molecular cloning of the gene of a penicillin-binding protein supposed to cause high resistance to $\beta$-lactam antibiotics in Staphylococcus cureus. Journal of Bacteriology 167, 975-80. 
National Committee for Clinical Laboratory Standards. (1985). Methods for Dilution Antimicrobial Susceptibility Tests for Bacteria that Grow Aerobically. Approved Standard M7-A. National Committee for Clinical Laboratory Standards, Villanova, PA.

Reynolds, P. E. (1985). Inhibitors of bacterial cell wall synthesis. In The Scientific Basis of Antimicrobial Chemotherapy (Greenwood, D. \& O'Grady, F., Eds), pp. 13-40. Cambridge University Press, Cambridge.

Reynolds, P. E. \& Brown, D. F. J. (1985). Penicillin-binding proteins of beta-lactam-resistant strains of Staphylococcus aureus. FEBS Letters 192, 28-32.

Reynolds, P. E. \& Fuller, C. (1986). Methicillin-resistant strains of Staphylococcus aureus, presence of identical additional penicillin-binding protein in all strains examined. FEMS Microbiology Letters 33, 251-4.

Song, M. D., Wachi, M., Doi, M., Ishino, F. \& Matsuhashi, M. (1987). Evolution of an inducible penicillin-target protein in methicillin-resistant Staphylococcus aureus by gene fusion. FEBS Letters 221, 167-71.

Tonin, E. \& Tomasz, A. (1986). $\beta$-Lactam-specific resistant mutants of Staphylococcus aureus. Antimicrabial Agents and Chemotherapy 30, 577-83.

(Received 29 July 1988; accepted 10 October 1988) 